\title{
ANALISE DOS TIPOS DE ANSIEDADE ENTRE JOGADORES TITULARES E RESERVAS DE FUTSAL
}

Daniel Bocchini

Lais Morimoto

Deise Rezende

Gilmara Cavinato

Luiz Marcelo Ribeiro da Luz

\section{Resumo}

objetivo do trabalho e analisar se existem diferenças no tipo de ansiedade apresentada entre os jogadores titulares e reservas. Foi analisado um grupo de adolescentes (16 e 17 anos, do sexo masculino) que praticam o futsal no clube Ypiranga e foi aplicado o teste CSAI-2 que verifica três tipos de ansiedade que sao: o cognitivo (estado emocional), somático (respostas fisiológicas) e autoconfianca (segurança em relação ao esporte). Os resultados mostraram que os jogadores titulares apresentaram prevalência de ansiedade somática enquanto os reservas apresentaram maior ansiedade cognitiva. Na autoconfiança ambos apresentaram um nível elevado. Portanto consideramos a importância de um trabalho multidisciplinar e individualizado para um melhor desempenho.

\section{Palavras-Chave}

Ansiedade; Futsal; Psicologia do Esporte.

\section{ANALYSIS OF THE ANXIETY TYPES BETWEEN TITULAR PLAYERS AND FUTSAL RESERVATIONS}

Daniel Bocchini

Lais Morimoto

Deise Rezende

Gilmara Cavinato

Ms Luiz Marcelo Ribeiro da Luz

\begin{abstract}
For the present study, a teenagers group was analyzed (males ranking from 16 to 17 years old) who plays indoor soccer in the Ypiranga Club, and a test CSAI-2, which verifies three kinds of anxiety, was applied. They are: the cognitive (emotional estate), the somatic (physiologic response) and selfconfident (security regarding the sport). The goal of this study is to analyze whether there are differences in the kind of anxiety that appears in the titular and the replacements players. The results showed that the titular players presented a high somatic anxiety while the reserve players presented a greater cognitive anxiety. In the self-confident both presented a high level. Therefore, we consider the importance of a multidisciplinary and individualized study for a better performance.
\end{abstract}

\section{Key-Words}

Anxiety; Indoor Soccer; Psychology of Sport. 


\section{INTRODUÇÃO}

O futsal e um esporte coletivo praticado por grande parte da população brasileira, devido a facilidade que oferece em relação a condições estruturais para que seja jogado.

Por se tratar de um fenômeno sociocultural o esporte e um dos conteúdos mais trabalhados na Educação Física, por isso, e alvo permanente de estudiosos e pesquisadores que buscam incessantemente decifrar suas múltiplas dimensões.

A Psicologia do Esporte e uma linha de pesquisa das Ciências dos Esportes, que procura achar respostas, ainda que não definitivas, para diagnosticar e intervir nos conteúdos específicos do esporte. Buriti (2001) relata que a psicologia do esporte tem inicio na década de vinte, mas este e um fenômeno que já vinha sido abordado de outras formas desde o final do século XVIII.

Dentro dessa linha de pesquisas da Psicologia do Esporte um dos temas mais estudados atualmente e a ansiedade, isto vem ocorrendo devido a diversos fatores inerentes ao esporte de alto rendimento que vêem influenciando significativamente o desempenho dos atletas. Situações de objetivos competitivos a alcançar, de desafios, de rivalidades, de expectativas, e de uma série de outros fatores associados, como por exemplo, de prestigio, de dinheiro, auto-estima, admiração social, reconhecimento entre outros.

"Todas estas situações acabam gerando, nos atletas, estados afetivos e somáticos complexos e inerentes as particularidades de cada competição e de cada personalidade. Essas vivencias esportivas, tendo em vista a personalidade de cada atleta e a carga afetiva que este coloca na competição, provocam reações emocionais atuais ou antecipatórias, tais como a ansiedade, estresse, medo, insegurança, depressão, angustia"(BALLONE, 2004).

A ansiedade e definida por Castillo (2000) como um sentimento vago e desagradável de medo, apreensão, caracterizado por tensão ou desconforto derivado de antecipação de perigo, de algo desconhecido ou estranho.

Andrade e Gorenstein (1998) também descrevem a ansiedade como um estado emocional com componentes psicológicos e fisiológicos, que faz parte do desenvolvimento do ser humano, podendo tornar-se patológica quando acontece de forma exagerada e sem uma situação real ameaçadora que a desencadeie. 
Ballone (2004) esclarece que e importante ter em mente que a principal diferença a ser considerada e em relacao ao estado de ansiedade momentânea, que e aquela fisiológica e necessária para o individuo se adaptar a uma determinada circunstancia e a ansiedade traço da personalidade, que e a característica de determinadas pessoas, naturalmente ansiosas.

Existem duas dimensões distintas para a ansiedade se apresentar. Segundo Spielberger apud De Rose Junior (1985), pode ser classificada como:

Ansiedade-traço: predisposição de uma pessoa perceber certas situações como ameaçadoras ou não, respondendo a elas com níveis variados de ansiedade-estado.

Ansiedade-estado: estado emocional imediato, caracterizado por um sentimento de medo e apreensão e tensão, acompanhado ou associado com a ativação do sistema nervoso autônomo.

No esporte, a ansiedade foi dividida em dois tipos, proposta por Martens apud Ballone, (2004); a ansiedade cognitiva e a ansiedade somática, as quais não teriam a mesma influencia no rendimento do atleta. Esse autor acha que o rendimento cairia quando a ansiedade cognitiva aumenta e, pelo contrario, melhoraria quando a ansiedade somática aumenta moderadamente, mas igualmente diminuindo quando esta ultima também e intensa.

Em termos de ansiedade somática, que surge no momenta da competição e envolve a participação do organismo como um todo, colocando-o em estado de alerta e resultando no aumento dos níveis de adrenalina e cortisol, e preferível que o atleta tenha um nível levemente superior ao nível normal durante a competição. A ansiedade somática e representada pela auto-percepção aos aspectos fisiológicos provenientes da ansiedade e esta diretamente relacionada com a ativação fisiológica causando respostas como: taquicardia, aumento da freqüência respiratória, dor de estômago, entre outras.

Um elevado nível de ativação através da ansiedade somática e essencial para as atividades globais que requerem rapidez, resistência física e forca, como por exemplo, nos jogos de futebol, basquete, lutas corporais, corridas, braço de ferro, natação, etc. Entretanto, um nível elevado de ansiedade somática seria negativo para habilidades complexas que exigem movimentos musculares finos, coordenação, concentração e equilíbrio. 
A ansiedade cognitiva por sua vez caracteriza-se como um componente mental da ansiedade-estado causado pelo medo de uma avaliação negativa, relacionando-se com pensamentos duvidosos a respeito de atingir um determinado objetivo, ou seja, expectativas e auto-avaliação, podendo causar reação como o medo, apreensão, apatia, desinteresse, pensamentos negativos, entre outros. Este tipo de ansiedade, quando aumentada, produziria efeitos negativos nas atividades esportivas que requerem maior concentração, estratégia e agilidade psicomotora fina, como por exemplo, no xadrez, sinuca, golf, tiro ao alvo, dança, etc.

Um terceiro aspecto relacionado a ansiedade somática e cognitiva que podem comprometer o desempenho dos atletas e o estado afetivo, que sua conseqüência mais direta e a autoconfiança. Que e definida por Brandão (2007) como a convicção que um atleta precisa ter para executar determinados comportamentos e alcançar com sucesso uma tarefa. A autoconfiança não se refere a habilidade do atleta em si, mas à avaliação que o atleta tem de sua própria habilidade, ou seja, na confiança que ele tem em sua capacidade para lidar com as necessidades do esporte.

\section{ANSIEDADE EM RELAÇÃO AO ESPORTE E COMPETIÇÃO}

A ansiedade-traço competitiva (ATC) e uma característica psicológica relativamente estável onde o atleta percebe certos estímulos do meio competitivo como ameaçadores ou não e a eles respondem com níveis variados de ansiedade-estado. A forma como o atleta interpreta esses estímulos pode variar de acordo com o tipo de esporte, idade e sexo.

A ansiedade afeta o desempenho do aluno ou atleta positiva e negativamente, devendo desta forma saber qual o nível de ansiedade que cada individuo deve alcançar para que este obtenha benefícios deste, caracterizado como desempenho ótimo. (BURM, 2001).

Frischnecht (1990) afirma que a ansiedade e um dos impeditivos mais comuns para um bom desempenho. Quando se tem um nível alto de ansiedade o "campo" de concentração de um individuo e totalmente atingido então ele começa a prestar atenção apenas a um número de sinais limitados, diminuindo assim seu desempenho.

A relação entre atleta e treinador também e muito importante, pois ambos passam por momentos de estresse. Contudo, o modo pelo qual eles enfrentam este nível de estresse reflete no modo pelo qual cada um vai conseguir lidar com as emoções e características individuais do outro (treinador/atleta), 
(CRATTY, 1983). Dessa forma, a preparação psicológica dos atletas ganha uma importância cada vez maior no âmbito esportivo.

Nesta perspectiva, o treinador deve ter uma função fundamental na formação, informação e reorientação global das atitudes dos pais dos atletas com quem trabalha. Deve atuar de forma a esclarecer a razão e o objetivo do programa desportivo, alem de manter expectativas realistas e ajuda-los a desenvolverem adequadamente suas próprias expectativas em função dos seus limites e potencialidades.

\section{A IDADE E A RELAÇÃO COM A ANSIEDADE}

Segundo Cratty (1983) a ansiedade pode se alterar conforma a faixa etária, apresentando maiores níveis durante os últimos anos da adolescência, e tendem a diminuir tais níveis aos 30 anos.

Os níveis elevados de ansiedade correspondem as idades em que, tanto homens quanta mulheres, atingem o ápice de seu potencial físico no esporte. No entanto, e altamente provável que os atletas que permanecem em um determinado esporte podem aprender a lidar melhor com a ansiedade situacional. Elevando assim a ansiedade a um nível ótimo, variando conforma a idade, personalidade e situação.

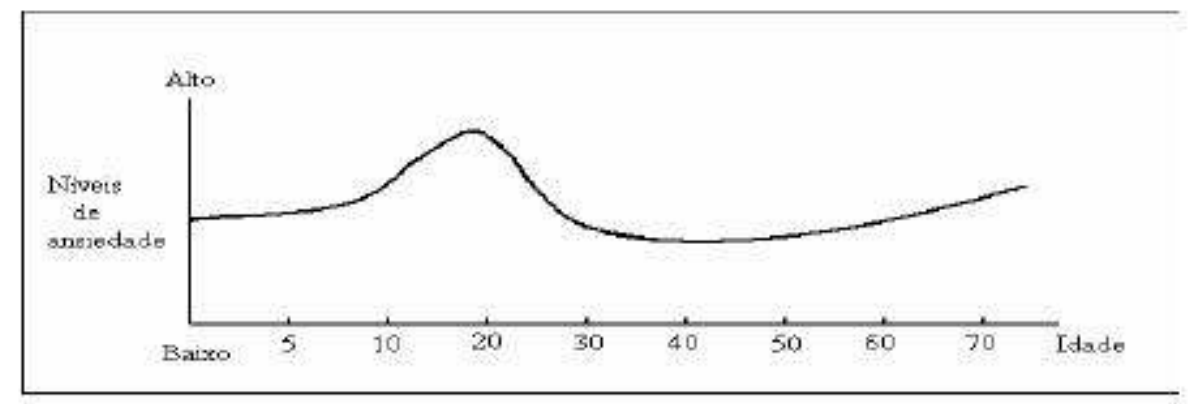

(CRATTY, 1983)

\section{METODOLOGIA}

Foi aplicado em 16 atletas o teste sobre ansiedade CSAI-2 que contem 27 perguntas nas quais os sujeitos optam por 1 = nada, 2 = alguma coisa, 3 = moderado e $4=$ muito, de acordo com as perguntas. Sendo referentes aos seus sentimentos durante a competição, resultando assim em um score da sua ansiedade cognitiva, somática e sua autoconfiança.

A pontuação do CSAI-2 e feita pela soma separada de três sub-escalas, com pontuação variando de 9 a 36. Para melhor compreensão e interpretação dos resultados, os níveis de ansiedade foram categorizados em baixo (entre 9 e 18 pontos), médio (entre 18 e 27 pontos), e alta(entre 27 e 36 pontos), de acordo com a somatória obtida através da analise dos questionários. 
Apos a pontuação, foi realizado comparação estatística entre os grupos pelo teste $t$ de student com variâncias equivalentes. Em todos os casos, foi adotado nível de significância de $\mathrm{p}<0,05$. Os gráficos obtidos são apresentados nos resultados e discussão.

\section{CARACTERIZAÇÃO DOS SUJEITOS}

O grupo escolhido foi a equipe de futsal masculino do Clube Atlético Ypiranga na cidade de São Paulo, estado de São Paulo, formado por 16 atletas do sexo masculino, na categoria sub-17, com idade entre 16 e 17 anos. A freqüência de treinos são de duas vezes por semana com duração de duas horas cada treino. O grupo trabalha ha cinco anos com a mesma formação.

A equipe e dividida em time titular, composto por cinco atletas, os números respectivos dos sujeitos são: $5,6,9,10,11$ e o time reserva são os demais atletas $1,2,3,4,7,8,11,12,13,14,15,16$.

\section{RESULTADOS E DISCUSSÃO}

A seguir são apresentados os gráficos obtidos do questionário CSAI- 2.

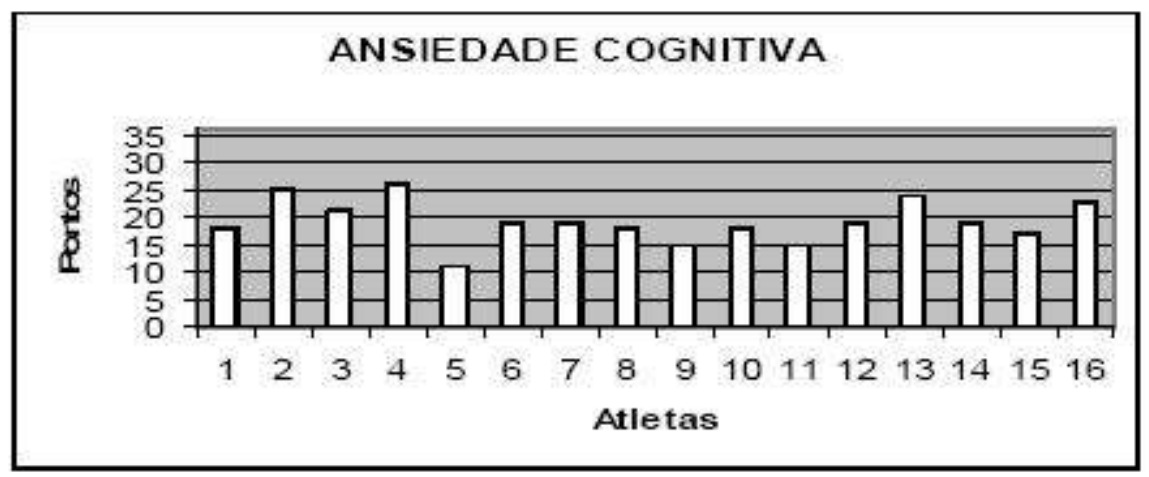

Grafico 1- Analise do nível de Ansiedade Cognitiva.

O score cognitivo apresentou uma media de 19,8 pontos com desvio padrão de 3,8. Esse dado aponta que os atletas possuem um nível de estado emocional elevado. Isto pode ser explicado pelo fato que estão sendo influenciados pelo ambiente, família, bom relacionamento do grupo e por estarem em época de competição. 


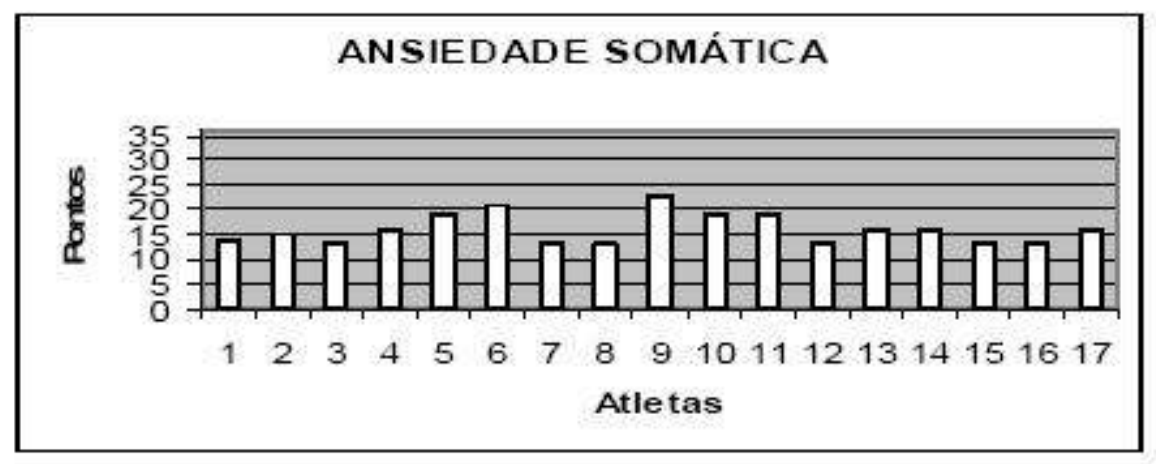

Grafico 2- Analise do nível de Ansiedade Somatica.

O score somático apresentou media de 16,3 pontos com desvio padrão de 3,1. Esse dado aponta que os atletas possuem um nível de respostas físiológicas abaixo da media esperada. Isto significa que os atletas podem estar menos suscetíveis a respostas fisiológicas.

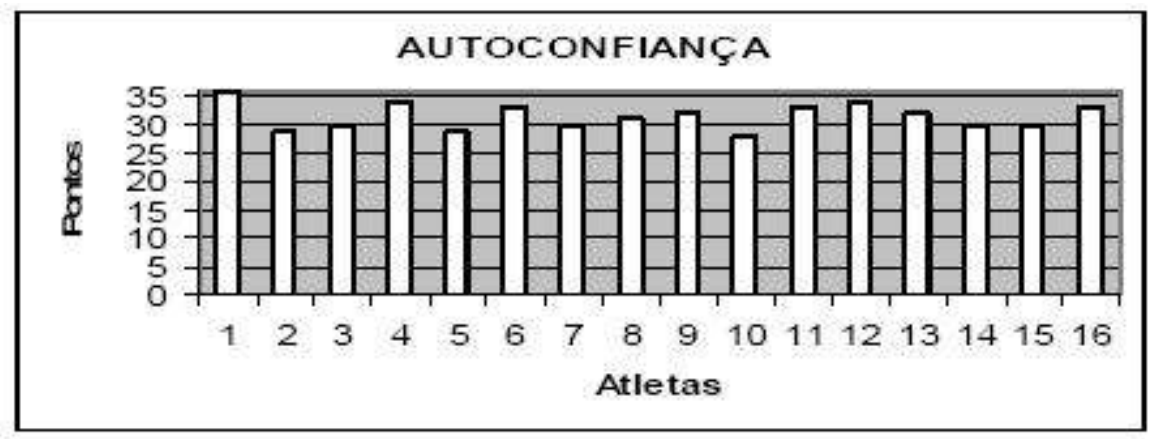

Gráfico 3 - Analise do nível de Ansiedade Autoconfiança.

O score da autoconfiança apresentou média de 31,5 pontos com desvio padrão de 2,2 como mostra os gráficos. Esse dado aponta que os atletas possuem um nível de autoconfiança elevado. Isto pode ser explicado pelo fato que os atletas se sentem confiantes provavelmente por possuírem um bom relacionamento dentro do grupo e com a comissão técnica. 


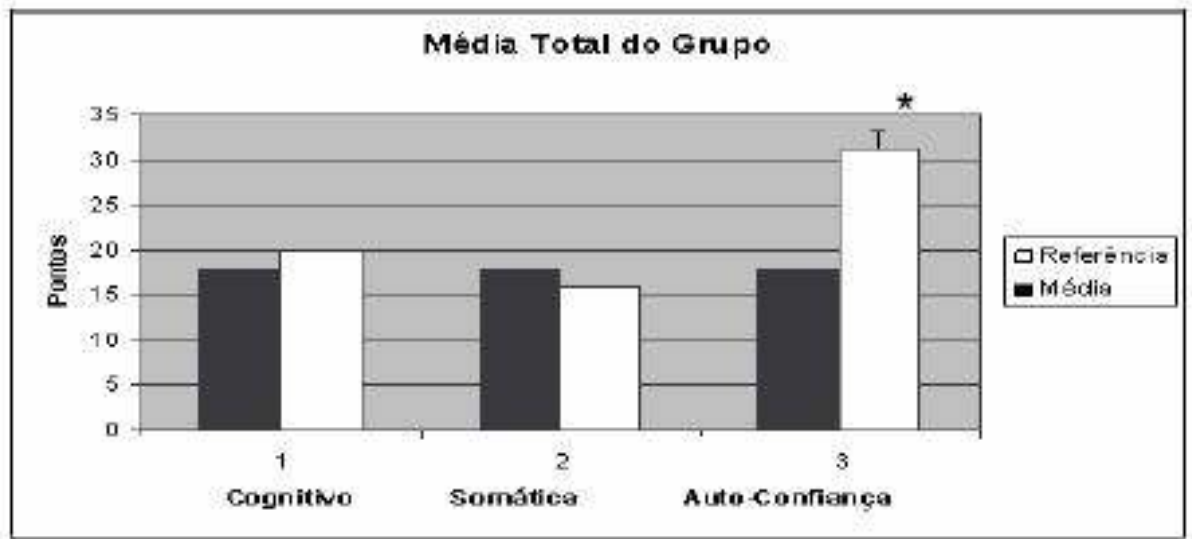

Gráfico 4- Análise da média geral do grupo

E possível notar uma significativa diferença (20 pontos) entre a referenda e o grupo de atletas perante a autoconfiança. Buriti (2001) afirma em seus estudos que isto pode ser justificado pelo fato de possuírem uma motivação elevada, equilíbrio emocional e segurança de um bom desempenho por estarem passando por uma fase de competições e principalmente por este ter uma coesão positiva entre os atletas e a comissão técnica.

Ambos os grupos apresentaram um acentuado score cognitivo, caracterizado por medo e apreensão de uma avaliação negativa de seu desempenho, entretanto como demonstrou um nível elevado de autoconfiança, a ansiedade cognitiva pode estar relacionada aos pensamentos e duvidas da consecução da vitória, ou a autoconfiança estar relacionada aos seus objetivos. O grupo obteve também um baixo score somático e uma alta concentração no score de autoconfiança.

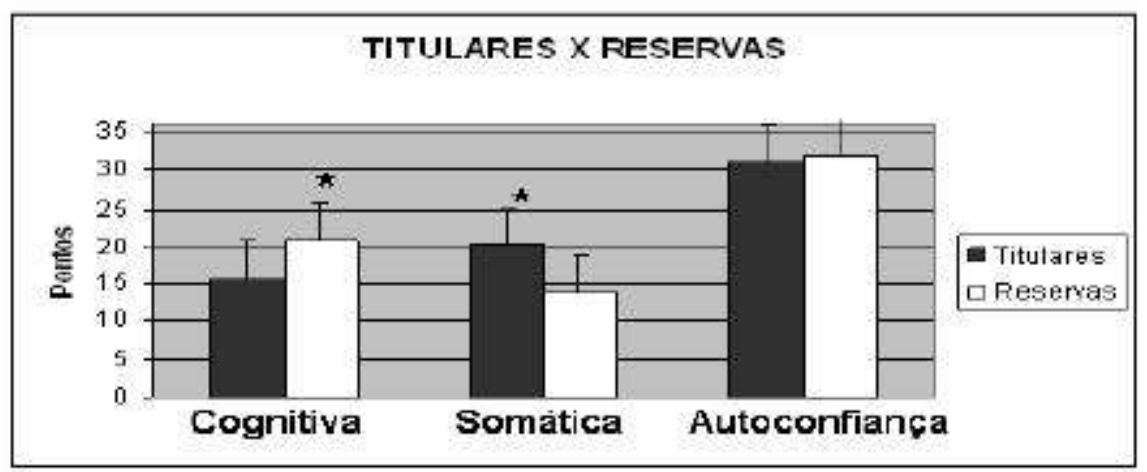

Gráfico 5 - Comparação entre o grupo do time titular e reserva na ansiedade cognitiva, somática e autoconfiança. 
No score cognitivo, a equipe titular apresentou uma media de 15,6 pontos com desvio padrão de 3 ,1. A equipe reserva apresentou uma media de 20 pontos com desvio padrão de 3,1 . Pode-se verificar que a equipe reserva possui maior nível emocional comparada a titular. Isto pode ser explicado por Buriti (2001) já que o mesmo afirma que o medo do fracasso de não saber as conseqüências podem alterar seu desempenho caso venham a jogar, já que estão sendo subestimados da sua capacidade por estarem na reserva.

No score somático, a equipe titular apresentou media de 20,2 pontos com desvio padrão de 1,7 . A equipe reserva obteve uma media de 13,8 com desvio padrão de 1,2.

Pela conseqüência dos indivíduos estarem no time titular, o score somático foi mais alto, ligado ao estado fisiológico (liberação de adrenalina e cortisol), caracterizado por um estado imediato da situação.

No score da autoconfiança, a equipe titular apresentou uma media de 31 pontos com desvio padrão de 2,3 e a equipe reserva apresentou uma media de 31,7 pontos com desvio padrão de 2,2. O grupo apresentou-se homogêneo, em relação a autoconfiança não mostrando diferença significativa entre os indivíduos, fator no qual pode ser direcionado pelo técnico e a forma que o time lhe da com as situações problemas e objetivos do time. Este dado pode ser explicado também por possuírem total confiança própria para realizar as tarefas e desafios que lhe serão propostos na fase final do campeonato. Um outro fator importante a ser mencionado e a idade, já que os atletas estão na faixa etária que caracteriza a adolescência. Conforme Cratty (1983) nesta fase apresentam maiores níveis durante os últimos dois anos, alem de atingirem o ápice de seu potencial físico no esporte.

\section{CONSIDERAÇÕES FINAIS}

Os resultados mostraram diferenças significativas entre os titulares e reservas nos níveis emocionais e fisiológicos (apresentados como cognitivo e somático respectivamente). Em relação a autoconfiança apresentaram o mesmo nível. De maneira mais especifica, na relação de nível emocional, os reservas apresentaram maiores resultados, uma possível hipótese e por estarem apreensivos por não saberem se irão jogar ou não e como será a sua performance.

No nível fisiológico, os titulares apresentaram maiores resultados conforme o esperado, já que estão jogando e a situação de jogo estimula a liberação de hormônios. 
E por último no nível de autoconfiança, ambos os grupos apresentaram alto nível assim, podemos verificar que os atletas estão confiantes para atingir seus objetivos relacionados à competição.

E necessário que haja um trabalho multidisciplinar para que os atletas tenham condições para manter um equilíbrio da ansiedade e de outros aspectos para que ocorra a melhoria do seu rendimento. É necessário também que uma intervenção de um psicólogo para realizar e aplicar atividades que intervenham nas dificuldades apresentadas.

\section{REFERENCIAS}

ANDRADE. L. H. S. G.; GORENSTEIN, C. Aspectos gerais das escalas de avaliação de ansiedade. Revista de Psiquiatria Clinica, São Paulo, v. 25, n. 6, nov./dez. 1998.

BALlONE, G. J. Ansiedade e esporte. Disponível em www.virtualpsv.org/temas/esporte.html>. Acesso em: 14 out. 2007.

BECKER JUNIOR, B. Manual de Psicologia do Esporte e Exercício. Porto Alegre: Novaprova, 2000.

URITI, M. A. Psicologia do esporte. Alínea, 2001.

CRATTY, B. J. Psicologia do esporte. Prentice Hall, 1983.

MUTTI, D. Futsal: da iniciação ao alto nivvel. 2. ed. Phorte, São Paulo: Phorte, 2003.

CASTILlO, A. R. R. et al. Transtornos de ansiedade. Revista Brasileira de Psiquiatria, São Paulo, v. 22, n. 2, 2000. São Paulo 2000

FILHO, M. G. B. ; MIRANDA, R. Aspectos psicológicos do esporte competitivo. Revista Treinamento Desportivo, Curitiba, v. 3, n. 2, p. 84, 1998.

JUNIOR, A. C. Q.; VICENTIM, J.; CRESPILHO, D. Relações entre ansiedade e psicologia do esporte. Revista Digital, Buenos Aires, n. 98, jul. 2006.

JUNIOR, D. R.; VASCONCELLOS, E. G. Ansiedade traço competitiva e atletismo: Um Estudo Com Atletas Infanto-Juvenis. Revista Paul. Educ. Fis., Sao Paulo, jul./dez. 1997-

LUIZ, A. M.; A. G. et al. Depressão, ansiedade e competência social em crianças obesas. Revista Estudo da psicologia, v. 10, n. 1, Natal, 2005. Disponível em: $<$ http://www. scielo. br/scielo. php?pid=S 1413294X2005000100005\&script $=$ sci arttext\&tlng $=$ pt $>$.

OKAZAKI, F. H. A. et al. A influencia da posição de titular ou reserva na de auto-estima de atletas de futsal brasileiro. Revista Digital, Buenos Aires, v. 10, n. 90, Nov. 2005. Disponível em: $<$ http://www.efdeportes.com/ef $>$ 


\title{
Daniel Bocchini
}

Universidade Estadual de Campinas - UNICAMP

\section{Lais Morimoto}

Universidade Metodista de Sao Paulo - UMESP

\section{Deise Rezende}

Universidade Metodista de Sao Paulo - UMESP

\section{Gilmara Cavinato}

Universidade Metodista de Sao Paulo - UMESP

\author{
Luiz Marcelo Ribeiro da Luz \\ Universidade Metodista de Sao Paulo - UMESP
}

\section{Referência do artigo:}

\section{ABNT}

BOCCHINI, D. et al. Analise dos tipos de ansiedade entre jogadores titulares e reservas de futsal. Conexões, v. 6, p. 522-532, 2008.

\section{APA}

Bocchini, D., Morimoto, L., Rezende, D., Cavinato, G., \& Luz, L. M. R. (2008) Analise dos tipos de ansiedade entre jogadores titulares e reservas de futsal. Conexões, 6, 522-532.

\section{VANCOUVER}

Bocchini D, Morimoto L, Rezende D, Cavinato G, Luz LMR. Analise dos tipos de ansiedade entre jogadores titulares e reservas de futsal. Conexões, 2008; 6: 522-532. 